\title{
Pelvic Floor Ultrasound under Particle Swarm Intelligent Optimization Algorithm in Preoperative and Postoperative Evaluation of Female Stress Urinary Incontinence
}

\author{
Hongbin Zhang $\mathbb{D},{ }^{1}$ Hezhou Li $\mathbb{D},{ }^{1}$ Xin Zhao $\mathbb{D},{ }^{2}$ Juan Wu $\mathbb{D},{ }^{1}$ Xiao Liang $\mathbb{D},{ }^{3}$ \\ and Haiyan Lu iD 1 \\ ${ }^{1}$ Department of Ultrasound, The Third Affiliated Hospital of Zhengzhou University, Zhengzhou 450000, Henan, China \\ ${ }^{2}$ Department of Radiology, The Third Affiliated Hospital of Zhengzhou University, Zhengzhou 450000, Henan, China \\ ${ }^{3}$ Department of Publicity, The Third Affiliated Hospital of Zhengzhou University, Zhengzhou 450000, Henan, China
}

Correspondence should be addressed to Hongbin Zhang; tianpeng191030@zzu.edu.cn

Received 3 August 2021; Revised 29 September 2021; Accepted 30 September 2021; Published 29 October 2021

Academic Editor: Gustavo Ramirez

Copyright ( 2021 Hongbin Zhang et al. This is an open access article distributed under the Creative Commons Attribution License, which permits unrestricted use, distribution, and reproduction in any medium, provided the original work is properly cited.

This study aimed to explore the application of pelvic floor ultrasound under particle swarm intelligent optimization algorithm in the preoperative and postoperative evaluation of female stress urinary incontinence (SUI) and provide a theoretical basis for clinical diagnosis. In this study, 90 patients with SUI were enrolled, which were randomly and equally assigned into a blank group (healthy physical examination), control group (perineal ultrasound), and experimental group (pelvic floor ultrasound based on particle swarm intelligence optimization algorithm). The ultrasonic image segmentation and processing were carried out by a particle swarm intelligence optimization algorithm. Patients with stress incontinence were classified as group A, and patients without stress incontinence were classified as group B. The results of previous surgical examinations were the standard to judge the accuracy of pelvic floor ultrasound diagnosis based on the swarm intelligence optimization algorithm. The accuracy of diagnosing stress UI in the experimental group was $90.38 \%$, which was significantly higher than that of the control group (54.31\%) and the blank group $(38.95 \%)(P<0.05)$. The formation percentage of the urethral internal orifice in the experimental group was $82.5 \%$, which was significantly higher than that of the control group (65.4\%) and the blank group (12.5\%), and there was a statistical difference among the groups $(P<0.05)$. In the resting state, the vertical spacing $y$ between the neck of the bladder and the edge of the pubis of patients in group B was greater than that in group B, the horizontal spacing $x$ between the neck of the bladder and the edge of the pubis was smaller than in the blank group, and there were statistical differences among the groups $(P<0.05)$. In the state of Valsalva, the vertical spacing $y$ between the neck of the bladder and the edge of the pubis of patients in group B was smaller than that in group B, the horizontal spacing $x$ between the neck of the bladder and the edge of the pubis was greater than that in group B. The distance of the bladder neck shifting downward was greater than that in group B, and there were statistical differences among the groups $(P<0.05)$. In short, the pelvic floor ultrasound based on the particle swarm intelligent optimization algorithm was accurate in the diagnosis of stress UI. The application of pelvic floor ultrasound in the diagnosis of UI provided image data objectively for clinical diagnosis and had a high application value.

\section{Introduction}

The female pelvic floor structure is complicated, and it consists of multiple parts together to form a support system, which includes muscle groups, ligament fascia, and nerves. They support each other and keep the pelvic floor organs such as the uterus, bladder, and rectum in their normal positions [1]. If the pelvic floor structure is degraded or damaged, it will cause weak support and lead to abnormal position and function of the pelvic floor organs. These are common clinically female pelvic floor dysfunction diseases. Female pelvic floor dysfunction diseases include pelvic floor organ shedding and urinary incontinence (UI). There are two most common symptoms: pelvic floor organ shedding 
and UI $[2,3]$. According to the etiology, UI is classified into multiple categories, such as stress urinary incontinence (SUI) and mixed UI [4]. SUI is the most common type of UI, which refers to accidentally passing from the ureteral orifice when the abdominal pressure suddenly rises (sneezing, coughing, laughing, exercising, etc.) [5]. Although SUI does not endanger the life of the patient, frequent urine leakage can cause social dysfunction in women, seriously affect their physiology and psychology, and seriously reduce women's quality of life [6]. Therefore, strengthening the education of medical workers and early diagnosis are the key to treating SUI [7].

There are two main ways to diagnose SUI at present, subjective symptoms and objective examinations. Subjective symptoms play an irreplaceable role in the diagnosis of female SUI. At the moment, the auxiliary diagnosis methods of female SUI mainly include urodynamic examination, magnetic resonance imaging (MRI) examination, and ultrasound examination [8]. MRI can distinguish the soft tissues of the female pelvic floor very well, but it is not suitable for patients with metal implants in the body. In addition, MRI is expensive and difficult to promote in clinics [9]. Although the ultrasound of the pelvic floor is inferior to MRI in terms of showing the soft tissues of the female pelvic floor, perineal pelvic ultrasound is a minimally noninvasive surgery, economical and practical. It can also show the "anatomical image" of the patient's pelvic floor through images and data and especially has better reproducibility for anterior pelvic measurements [10]. In addition, many studies showed that pelvic floor ultrasound is a very critical link in the treatment evaluation of SUI patients before and after surgery. Therefore, perineal pelvic floor ultrasound has been widely used in the diagnosis of female SUI patients and has become a research central issue in recent years.

Particle swarm optimization (PSO) is an evolutionary computation, which was proposed by Dr. Eberhart and Dr. Kennedy in 1995. Based on the observation of animal cluster activity behavior, individuals in the group share information to make the movement of the whole group evolve from disorder to order in the problem-solving space, to obtain the optimal solution [11]. The multiobjective optimization problem of PSO exists in all fields of society, and it plays an increasingly important role in the optimization of medical images. The use of particle swarm intelligence algorithm to solve the multiobjective optimization problem in medical images is a central issue of current research. With its advantages, the PSO algorithm has been widely used in the multiobjective optimization solution of medical images after improvement [12]. The pelvic floor ultrasound based on swarm intelligence algorithm was used in the research to evaluate its application effect on preoperative and postoperative of female SUI and to provide some reference for the later research of this disease.

\section{Materials and Methods}

2.1. Clinical Data. In this research, 90 female patients were collected in the hospital from July 30, 2019, to March 30, 2020, and 90 patients were randomly divided into blank group $(n=30)$, control group $(n=30)$, and experimental group $(n=30)$. Health examination, perineal ultrasound, and pelvic floor ultrasound based on particle swarm intelligence optimization algorithm were used for diagnosis, and ultrasonic image segmentation and processing were carried out by particle swarm intelligence optimization algorithm [13]. Afterward, patients with SUI were recorded as group A, patients without SUI were recorded as group B, and they were compared and analyzed. The patient's age ranged from 22 to 60 years and the average age was $(49 \pm 8.28)$ years. The main symptoms of the patient were uncontrolled urine overflow when the abdominal pressure changed due to cough, exercise, and laughter. Clinically, it was learned from the patient that the patient can control the urine in the supine position, but patients may experience uncontrolled urine discharge when standing, and patients with severe conditions may also experience uncontrolled urine discharge when lying down. Figure 1 shows the normal anatomy of the urinary system. This research topic had been approved by the ethics committee of the hospital, and all patients participating in this research signed an informed consent form for the operation.

Inclusion criteria were as follows: (1) patients who experienced involuntary urine discharge when abdominal pressure increased (coughing, laughing, etc.), (2) patients who had undergone clinical pathological examination and met SUI symptoms, and (3) patients with no history of mental illness and normal communication.

Exclusion criteria were as follows: (1) patients who cannot finish the examination completely (health physical examination, perineal ultrasound examination, and pelvic floor ultrasound examination); (2) patients with pelvic floor organ prolapse (excluding cystocele); (3) patients who took diuretics, hormones, and other drugs in the three months before medical attention; (4) patients with a history of disease treatment before diagnosis and treatment; (5) patients who took estrogen drugs for a long time; (6) patients with a history of pelvic surgery or space-occupying disease; (7) patients with urinary system infection or other diseases; (8) patients with unclear pelvic floor ultrasound images stored in the data; and (9) patients with severe SUI, because patients with very severe SUI were rare.

\subsection{Ultrasound Inspection Methods. The Mindray Medical} DC-7 Doppler ultrasound diagnostic instrument was selected. The abdominal probe was a convex array probe, and the probe frequency was $2.5 \mathrm{MHz}$ and $5.0 \mathrm{MHz}$. The patient needed to empty the bladder before the examination, and the patient needed to be in the horizontal lithotomy position during the examination. The doctor applied the coupling agent to the instrument and reapplied it again with medical gloves. The 2D mode was selected and a medium pressure probe was placed between the vaginal orifice and the vaginal urethral orifice. The two sides of the vaginal labia majora were separated, in resting mode, the image was obtained by scanning the lines behind the median sagittal position. The patient remained horizontal during the examination to ensure that the central axis of the probe was parallel to the 


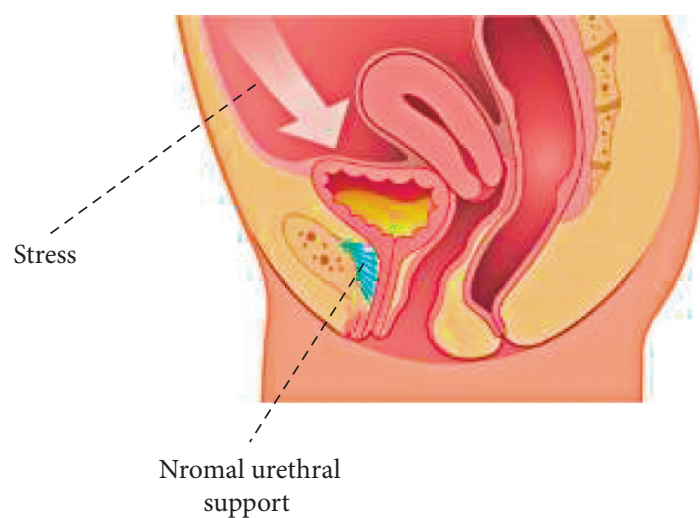

Figure 1: Normal anatomy of the urinary system.

horizontal line and the still picture or ultrasonic images were captured for later measurement and analysis. The probe should not be squeezed during the inspection to ensure that the pelvic floor organs were moved and reduce the rate of misdiagnosis. To ensure data consistency, the audit process and detection of images were performed by one person.

2.3. Construction of Particle Swarm Algorithm. The particle swarm algorithm was inspired by the swarm behavior of birds, fish schools, and so on and was proposed by Kenndey and Eberhart. Scientists took the predatory behavior of birds as an example. A flock of birds was looking for food in space. There was only one food source in this space; no bird knew the location of the food source, but all birds knew the distance among themselves and their food. In this case, the most direct and effective way for birds to find food was by searching the space around the birds closest to the food source. Scientists got inspiration from this behavior and then proposed the particle swarm algorithm. The POS algorithm started from a set of randomly initialized particles, and mainly updated the position of the particles step by step to obtain the optimal solution. In each iteration, particles can update their particle solutions by tracking two extreme positions, one was the best position in the individual history (Abest), and the other was the best position in the whole (Bbest). The mathematical description of the POS algorithm is shown in Figure 2.

It was assumed that $O$ is the number of particles in the population, $U$ is the dimension of the range space, and the position of the particle in each range space is represented as

$$
y=y_{t}^{1}, y_{t}^{2}, y_{t}^{3} \cdots y_{t}^{n} .
$$

The best position in the individual particle is represented as

Abest $_{t}=\left(\right.$ Abest $_{t}^{1}$, Abest $_{t}^{2}$, Abest $_{t}^{3} \cdots$ Abest $\left._{t}^{n} \quad t=1,2, \cdots n\right)$.

The best position in the whole is represented as

$$
\text { Bbest }=\left(\text { Bbest }_{1}, \text { Bbest }_{2}, \cdots \text { Bbest }_{n}\right) \text {. }
$$

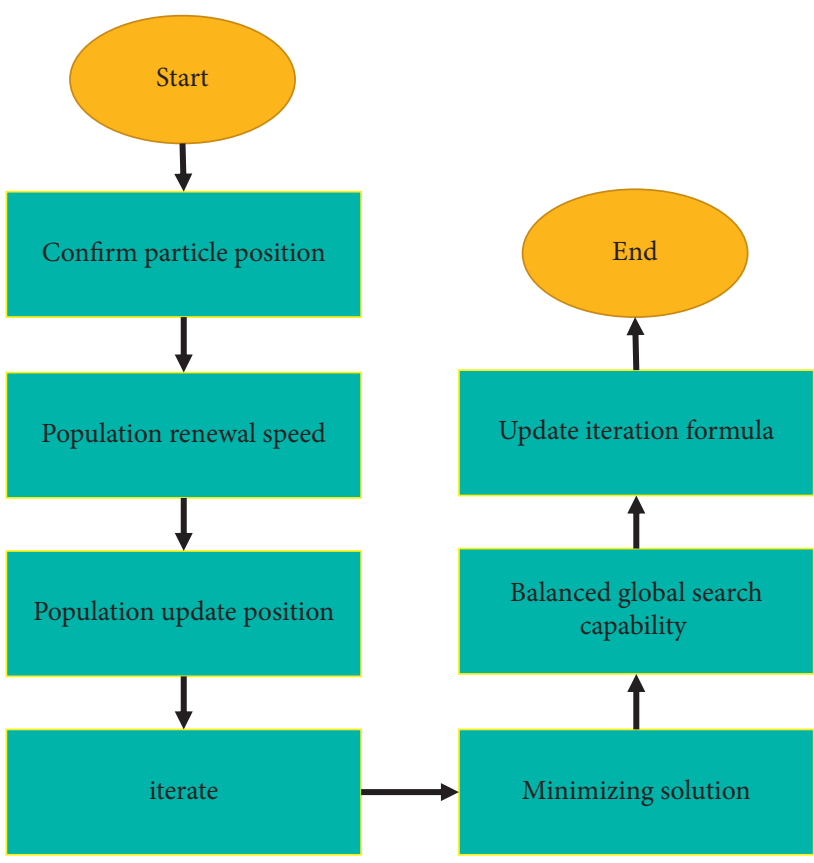

FIGURE 2: PSO algorithm.

The equation for the new velocity and position of the population are shown follows:

$$
\begin{aligned}
W_{t}^{k}(g+1)= & W_{t}^{k}(g)+b_{1} \cdot f_{1 k}(g) \cdot\left(\text { Abest }_{t}^{k}(g)-y_{t}^{k}(g)\right) \\
& +b_{2} \cdot f_{2 k}(g) \cdot \text { Bbest }_{t}(g)-y_{t}^{k}(g) \\
& y_{t}^{k}(g+1)=y_{t}^{k}(g)+y_{t}^{k}(g+1) .
\end{aligned}
$$

In equations (4) and (5), $t=1,2, \cdots n$ represent the index of the particle, $k=1,2, \cdots n$ is the spatial dimension of the particle solution, $g=1,2, \cdots N$ is the number of iterations, and $N$ is the maximum number of iterations. In order not to lose generality, the problem is minimized and solved. The updated equation is as follows:

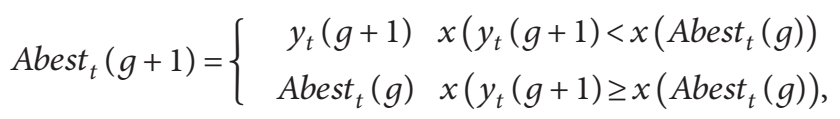

$$
\text { Bbest }(g+1)=\arg \min \left(x\left(\text { Abest }_{t}(g+1)\right) .\right.
$$

Because the original PSO algorithm did not solve the optimization problem very well, scholars and experts proposed an optimized POS algorithm. In the following equation, $\psi$ is the weighted weight, which mainly balances the global and local search capabilities of the algorithm. As the number of iterations increases, $\psi$ gradually decreases:

$$
\psi(g)=\psi_{\max }-\frac{g}{G} \cdot\left(\psi_{\max }-\psi_{\min }\right) .
$$

In equation (8), $\psi_{\max }$ is the maximum weight and $\psi_{\min }$ is the minimum weight. The maximum weight is usually set to 


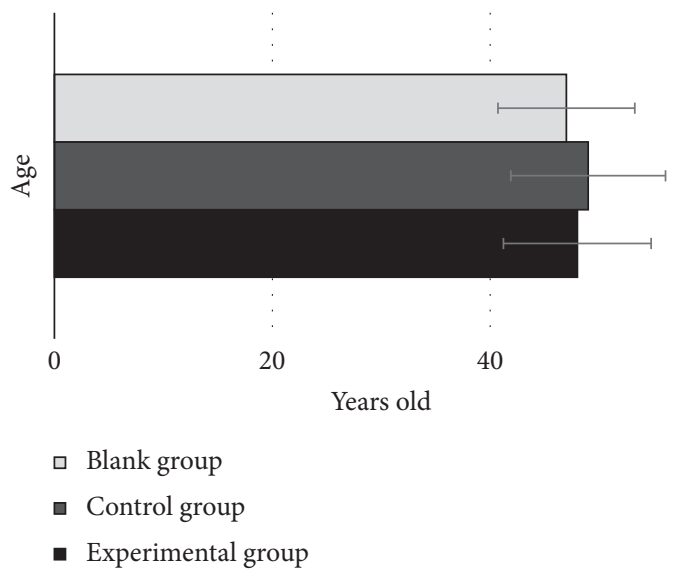

(a)

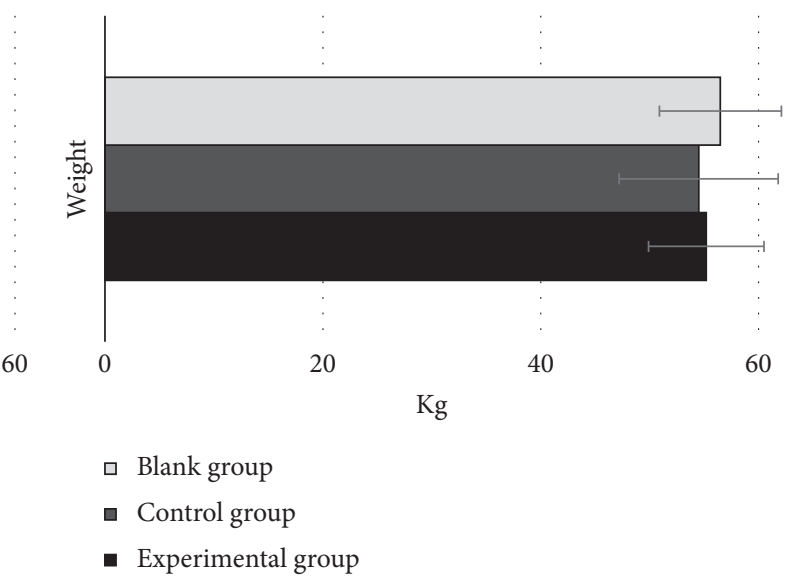

(b)

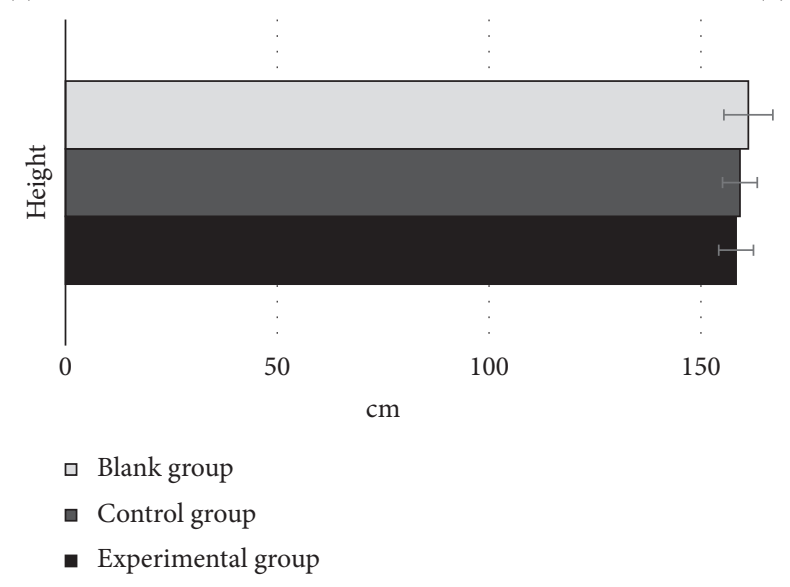

(c)

Figure 3: Difference of patients' general data. (a) Age, (b) weight, and (c) height.

0.9 , and the minimum weight is usually set to 0.4 . Therefore, the iterative equation for velocity is updated as

$$
\begin{aligned}
W_{t}^{k}(g+1)= & \psi(g) \cdot W_{t}^{k}(g)+b_{1} \cdot f_{1 k}(g) \cdot\left(\text { Abest }_{t}^{k}(g)-y_{t}^{k}(g)\right) \\
& +b_{2} \cdot f_{2 k}(g) \cdot \text { Bbest }_{t}(g)-y_{t}^{k}(g) .
\end{aligned}
$$

2.4. Observation Indicators. The indicators of patients were compared, including parameters under different conditions, mobility of bladder neck, and rotation angle of the urethra. The levels of BND, URA, and PVUA in the three groups were compared [14]. BND referred to the distance between the neck of the bladder and the inferior edge of the pubis in the resting state URA referred to the difference in urethral inclination between the quiescent state and the Valsalva state. PVUA referred to the angle between the posterior wall of the bladder and the proximal urethra in the Valsalva state.

2.5. Statistical Processing. The data processing of the research was analyzed by SPSS 19.0 version statistical software. The measurement data were expressed by the mean \pm standard deviation $(\overline{\mathrm{x}} \pm s)$, the count data was expressed by the percentage (\%), and $t$-test, $\chi 2$ test, and so on were used. The treatment success rate, second operation rate, and complications of the conventional group and the intervention group were tested by variance test. $P<0.05$ was considered statistically different among groups.

\section{Results}

3.1. Difference of Patients' Basic Data. The basic data difference of the three groups showed that the mean age of the three groups was $(49 \pm 8.28)$ years. In the blank group, the mean age of patients was $(48 \pm 6.28)$ years, the mean weight of patients was $(55.2 \pm 5.6) \mathrm{kg}$, and the mean height of patients was $(158.3 \pm 5.78) \mathrm{cm}$. In the control group, the mean age of patients was $(49 \pm 7.1)$ years, the mean weight of patients was $(54.5 \pm 7.3) \mathrm{kg}$, and the mean height of patients was $(159.2 \pm 4.1) \mathrm{cm}$. In the experimental group, the mean age of patients was $(47 \pm 6.78)$ years, the mean weight of patients was $(56.5 \pm 5.3) \mathrm{kg}$, and the mean height of patients was $(161.2 \pm 4.1) \mathrm{cm}$. There was no statistical difference in age, height, and weight of patients in the three groups, and the comparison among groups was feasible $(P>0.05)$, as shown in Figure 3. 


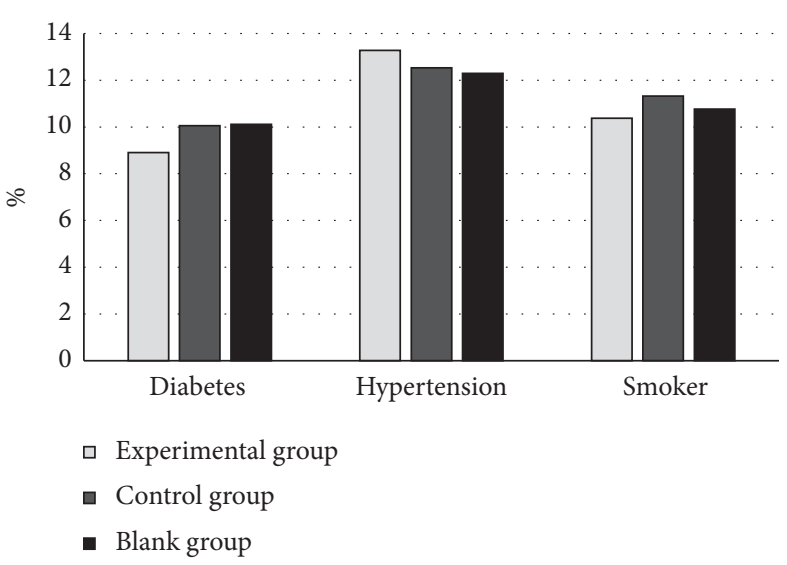

(a)

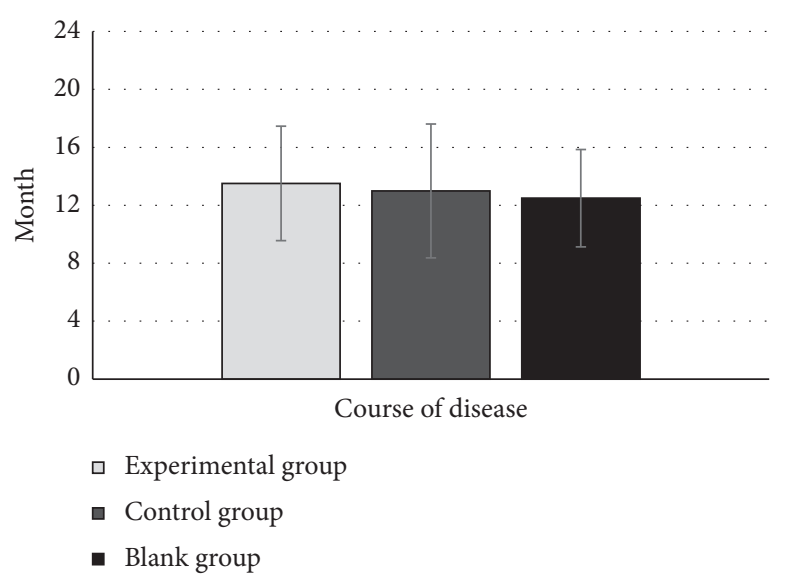

(b)

FIGURE 4: Comparison of (a) the history of diabetes, hypertension, and smoking and (b) the course of disease among the three groups of patients.

In Figure 4, there is no significant difference in the history of diabetes, hypertension, and smoking and course of disease among the three groups of patients $(P>0.05)$.

Figures 5-7 show the results of ultrasound examination of a 48-year-old female patient diagnosed based on swarm intelligence algorithm. The image quality after the PSO algorithm was relatively better, the noise was reduced, and the sharpness was obviously improved.

3.2. Algorithm Performance Comparison. The genetic algorithm and the traditional PSO were introduced and compared with the algorithm constructed in this research, and the sensitivity, specificity, and accuracy of the three algorithms for the diagnosis of patients under the basin ultrasound were calculated. Figure 8 shows that the sensitivity, specificity, and accuracy of the constructed algorithm for patient diagnosis were significantly higher than the genetic algorithm and the traditional particle swarm algorithm, with significant differences $(P<0.05)$.

\subsection{Difference of Diagnostic Accuracy of Patients in Each} Group. The ultrasound images of patients were diagnosed based on the swarm intelligence algorithm, as shown in Figure 9. The diagnostic accuracy of SUI in the experimental group was $90.38 \%$, which was significantly higher than that of the control group (54.31\%) and the blank group (38.95\%). There were statistical differences among the groups $(P<0.05)$. The formation rate of the urethral orifice was diagnosed based on the swarm intelligence algorithm. The urethral orifice formation percentage in the experimental group was $82.5 \%$, which was significantly higher than that in the control group (65.4\%) and the blank group (12.5\%). There was a statistical difference between the two groups $(P<0.05)$.

\subsection{Difference of Patients' Bladder Neck Mobility and Urethral} Rotation. Figure 10 shows that the movement of the bladder neck of group A was $(20.78 \pm 1.87)^{\circ}$, and the rotation of the

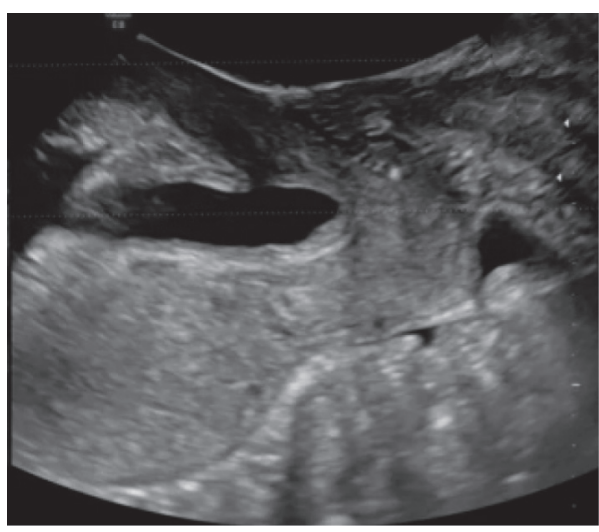

FIGURE 5: Schematic diagrams of the median sagittal view of the patient's two-dimensional ultrasound pelvic floor.

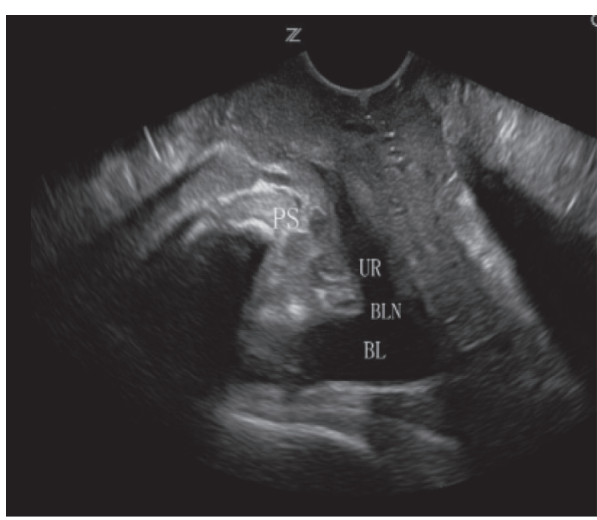

FIgURE 6: Sagittal view of the patient's pelvic floor (PS: pubic symphysis; UR: urethra; BL: bladder; BLN: bladder neck).

urethra was $(40.57 \pm 2.08)^{\circ}$. The movement of the bladder neck of the group B was $(11.82 \pm 1.02)^{\circ}$, and the rotation of the urethra was $(28.18 \pm 1.73)^{\circ}$. The difference between the two groups was obvious, and there was a statistical difference between the two groups $(P<0.05)$. 


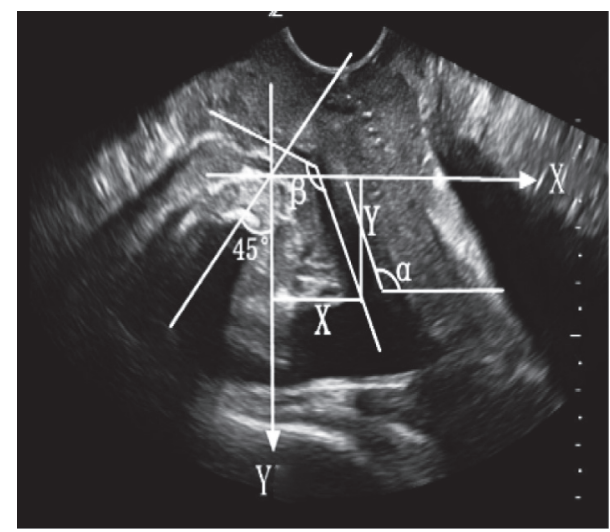

Figure 7: Pelvic floor transperineal ultrasound measurement of patients (x: the position of the bladder neck on the $x$-axis; $y$ : the position of the bladder neck on the $y$-axis; $\alpha$ : the posterior angle of the bladder urethra; $\beta$ : the angle formed by the proximal urethra and the distal urethra).

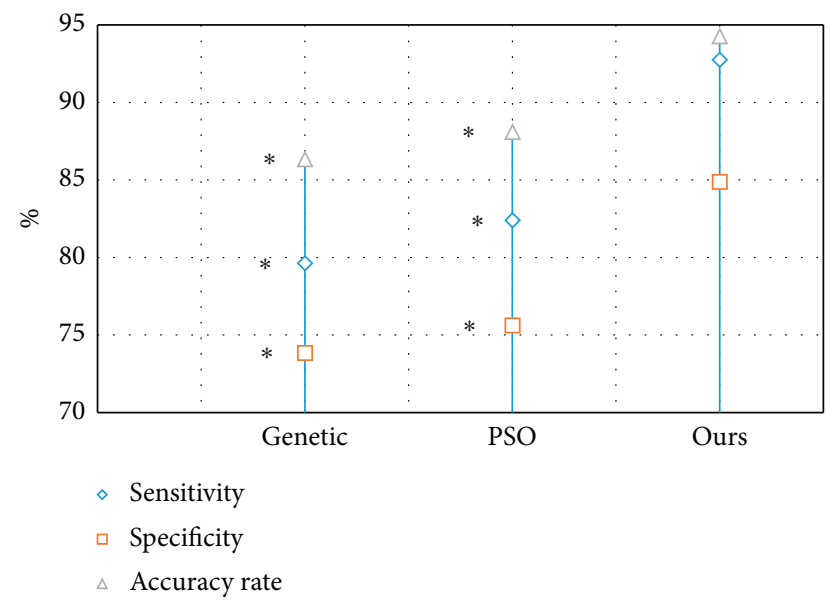

Figure 8: Algorithm performance comparison. Note. ${ }^{*}$ represents a significant difference compared with the algorithm proposed in this research $(P<0.05)$.

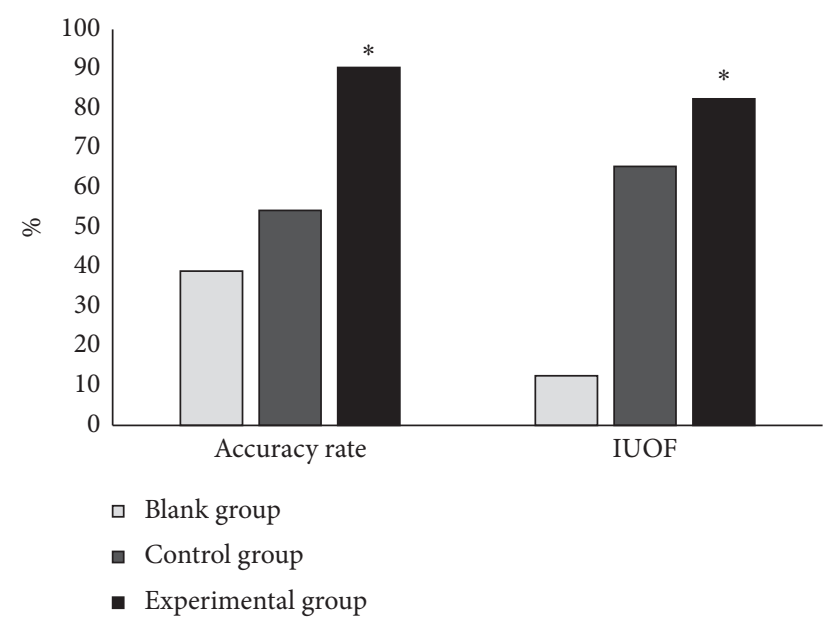

Figure 9: Difference of accuracy of examination results of patients. * indicates that there is a statistical difference between the experimental group and other groups $(P<0.05)$.

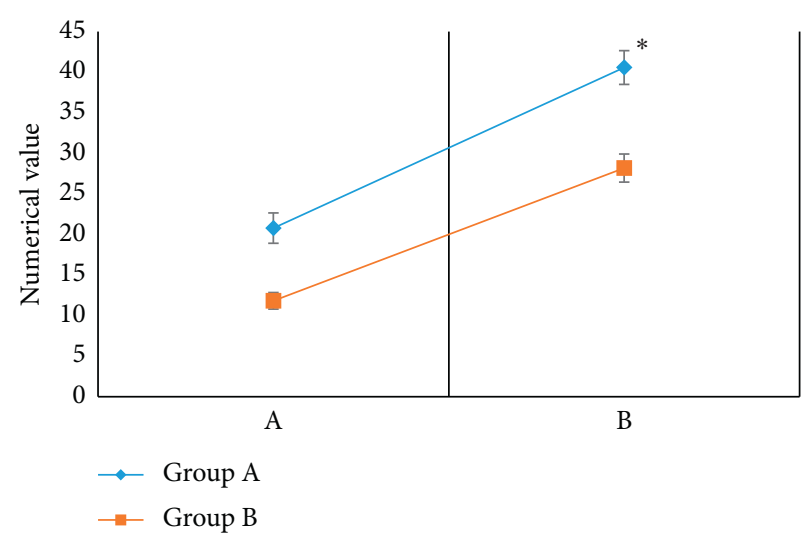

FIGURE 10: Difference of patients' bladder neck mobility and urethral rotation. A is the movement angle of the bladder neck; B is the rotation angle of the urethra. ${ }^{*}$ indicates that the difference between group A and group B is statistically remarkable $(P<0.05)$.

3.5. Difference of Ultrasound Parameters of Patients under Different States. Figure 11 shows that there are considerable differences between group $\mathrm{A}$ and group $\mathrm{B}$ in URA and PUVA under maximum Valsalva state $(P<0.05)$. There is no obvious difference in UIA in the resting state between the two groups $(P>0.05)$.

Figure 12 shows that, in the resting state, the bladder neck and the vertical spacing $y$ between the neck of the bladder and the edge of the pubis in group $\mathrm{B}$ is greater than that in group B, and the horizontal spacing $x$ between the neck of the bladder and the edge of the pubis is smaller than that in the blank group. There are statistical differences between groups $(P<0.05)$. Figure 13 shows that, in the state of Valsalva, the vertical spacing $y$ between the neck of the bladder and the edge of the pubis of patients in group $B$ is smaller than that in group B, the horizontal spacing $x$ between the neck of the bladder and the edge of the pubis is greater than that in group $\mathrm{B}$. The distance of the bladder neck shifting downward is smaller than that in group $\mathrm{A}$, and there are statistical differences between the groups $(P<0.05)$. The distance of the bladder neck down (BND) value is smaller than that of group $\mathrm{A}$, and there is a statistical difference between the groups $(P<0.05)$.

\section{Discussion}

UI is a gynecological disease with a high incidence in middle-aged and elderly women. SUI is one of the more common types. The incidence in middle-aged and elderly women is over 55\%, and there is still a part of untreated women with SUI, so the incidence of the disease is actually higher. SUI has become one of the main reasons for women's poor quality of life and impaired physical and mental health, and it is urgent to find a solution. To analyze the application effect of pelvic floor ultrasound in SUI before and after surgery, an optimization intelligence algorithm based on particle swarm was proposed in the research, which was compared with health physical examination and perineal ultrasound examination. The ultrasonic images of patients were diagnosed based on swarm intelligence algorithm, and 


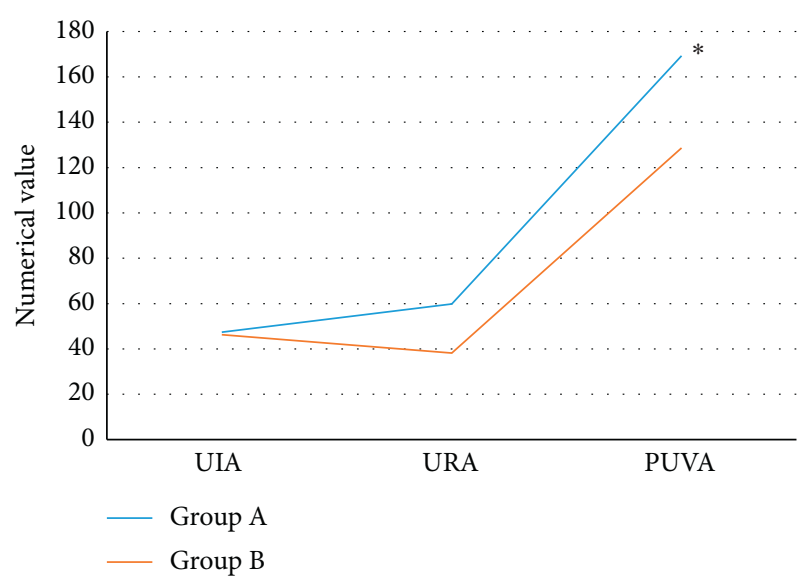

FIgURE 11: Difference of patients' bladder neck mobility and urethral rotation. ${ }^{*}$ indicates that there is a statistical difference between group A and group B $(P<0.05)$.

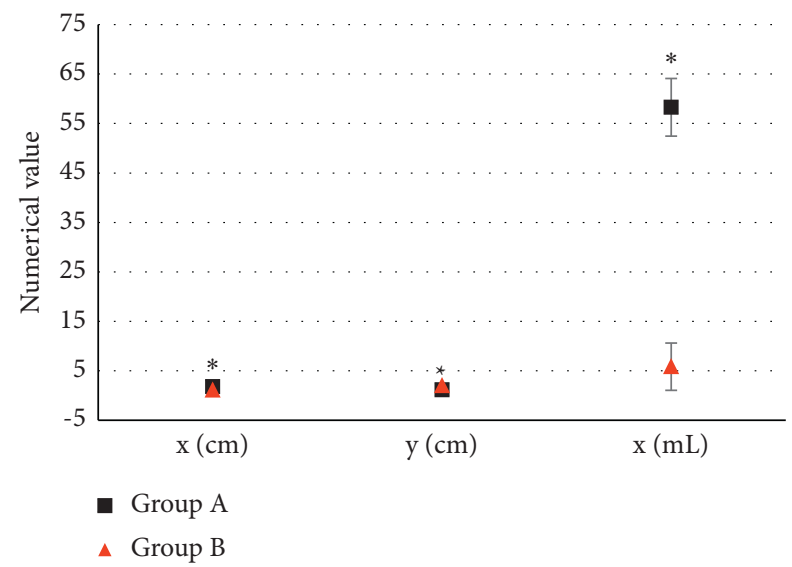

FIGURE 12: Difference of ultrasound parameters of patients at rest. $x$ and $y$ refer to the horizontal and vertical distance from the bladder neck to the lower edge of the pubic bone, respectively; $c$ represents the residual urine volume of the bladder. ${ }^{*}$ indicates that the difference between group A and group B is statistically dramatic $(P<0.05)$.

the results showed that the diagnostic accuracy of SUI in the experimental group was $90.38 \%$, obviously higher than that in the control group (54.31\%) and the blank group (38.95\%), and there was a statistical difference among the groups $(P<0.05)$. The urethral orifice formation rate of patients was diagnosed based on the swarm intelligence algorithm, and the urethral orifice formation percentage of patients in the experimental group was $82.5 \%$, which was significantly higher than that in the control group (65.4\%) and the blank group (12.5\%). The difference was statistically notable $(P<0.05)$. The conclusion of the research was similar to that of Brooks et al. [15], both of which indicated that the improved POS algorithm can significantly improve the accuracy of pelvic floor ultrasound diagnosis and the urethral orifice formation rate of patients. It had certain advantages and was applied to the examination and diagnosis of ultrasound. Studies showed that the movement difference between the front and back walls of the proximal urinary

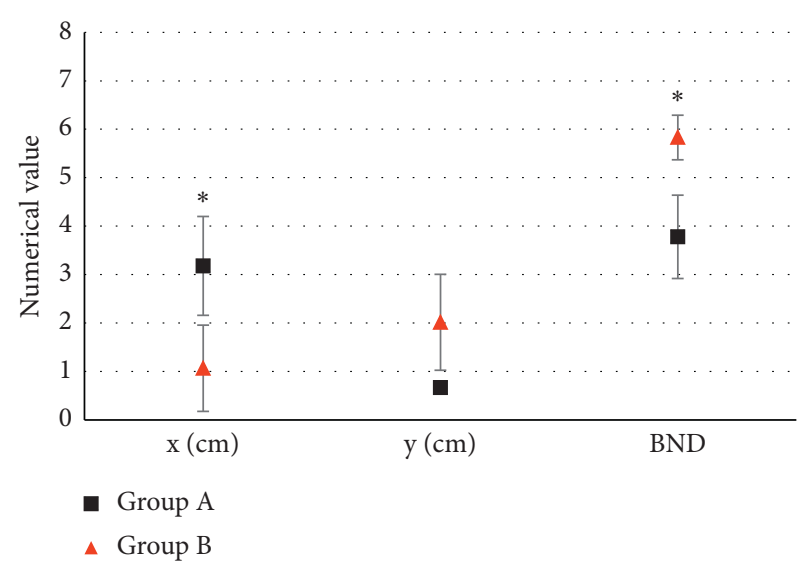

FIgURE 13: Analysis of ultrasound parameters of patients in the Valsalva state. $x$ and $y$ refer to the horizontal and vertical distance from the bladder neck to the lower edge of the pubic bone, respectively; BND means the bladder neck down distance. ${ }^{*}$ indicates that the difference between group A and group B is statistically substantial $(P<0.05)$.

tract formed a bladder neck funnel when the abdominal pressure of patients with UI increased. The percentage of bladder neck funnel formation of patients with SUI ranged from $18.6 \%$ to $97.6 \%$. Yin et al. [16] proposed in the research that judging whether the bladder neck funnel was formed was one of the criteria for diagnosing pelvic floor ultrasound and evaluating SUI in 2017. The diagnostic sensitivity, specificity, and accuracy of the proposed algorithm were significantly higher than those of genetic algorithm and traditional PSO $(P<0.05)$, indicating that the proposed algorithm could effectively improve the diagnostic effect of basin ultrasound for patients with SUI, and the improvement degree was superior to the traditional algorithm.

According to the movement of bladder neck and rotation of urethra, the movement of bladder neck in group A was $(20.78 \pm 1.87)^{\circ}$, and the rotation of urethra was $(40.57 \pm 2.08)^{\circ}$. In group $B$, the movement of the bladder neck was $(11.82 \pm 1,02)^{\circ}$, and the rotation of urethra was $(28.18 \pm 1.73)^{\circ}$. The difference between the two groups was obvious $(P<0.05)$. In the resting state, the vertical spacing $y$ between the neck of the bladder and the edge of the pubis in group B was greater than that in group B, and the horizontal spacing $x$ between the neck of the bladder and the edge of the pubis was smaller than that in the blank group. There were statistical differences between groups $(P<0.05)$. In the state of Valsalva, the vertical spacing $y$ between the neck of the bladder and the edge of the pubis of patients in group B was smaller than that in group $\mathrm{B}$, the horizontal spacing $x$ between the neck of the bladder and the edge of the pubis was greater than that in group B. The distance of the bladder neck shifting downward was smaller than that in group $\mathrm{A}$, and there were statistical differences between the groups $(P<0.05)$. The distance of the bladder neck down (BND) value was smaller than that of group $A$, and there was a statistical difference between the groups $(P<0.05)$. This was highly similar to the research results of Braekken et al. [17], which was because the pelvic floor structure of patients with SUI did not change alone. However, it was obvious that 
changes occurred when abdominal pressure increased, so UIA cannot be used as an indicator to evaluate women's SUI. A study by Nyhus et al. [18] found that, in the Valsalva state, the PUVA of SUI patients was much larger than that of normal women. In the resting state, there was no obvious difference in PUVA between the two groups of patients. Subsequently, through the comparison of 20 healthy women with 20 women with SUI, Martan et al. [19] found that PUVA of healthy women was significantly lower than that of SUI patients in resting and Valsalva states, and ultrasound indicated poor urethral-bladder junction support when PUVA value was too large.

\section{Conclusion}

To analyze the application effect of pelvic floor ultrasound before and after SUI, an optimization intelligence algorithm based on particle swarm was conducted in the research, which was compared with health physical examination and perineal ultrasound examination. It was also applied to the pelvic floor ultrasound examination of patients with SUI. The results showed that pelvic floor ultrasound based on particle swarm intelligence optimization algorithm significantly improved the diagnostic accuracy of SUI patients. The evaluation indicators based on pelvic floor ultrasound were used for the diagnosis of SUI. The rate of bladder neck funnel formation, URA, and BND were used for the diagnosis of SUI, and reliable and detailed data support were provided for clinical diagnosis. However, the long-term follow-up of patients was not implemented, and there was a lack of longterm index data, so we were unable to analyze the diagnostic value of basin ultrasound in long-term rehabilitation of patients with post-op SUI. In addition, the small sample size of patients may have some influence on the results of the study, so it should consider increasing the number of patients to further analyze the examination performance of ultrasound. In conclusion, this study provides data support for preoperative and postoperative evaluation of women with SUI.

\section{Data Availability}

The data used to support the findings of this study are available from the corresponding author upon request.

\section{Conflicts of Interest}

The authors declare no conflicts of interest.

\section{Acknowledgments}

This work was supported by the Medical Science and Technology Research Plan of Henan Province (no. LHGJ20190378).

\section{References}

[1] G. Capobianco, M. Madonia, S. Morelli et al., "Management of female stress urinary incontinence: a care pathway and update," Maturitas, vol. 109, pp. 32-38, 2018.
[2] B. Padilla-Fernández, G. M. Ramírez-Castillo, D. HernándezHernández, and D. M. Castro-Díaz, "Urodynamics before stress urinary incontinence surgery in modern functional urology," Eur Urol Focus, vol. 5, no. 3, pp. 319-321, 2019.

[3] A. Edenfield, R. Patnam, and S. Swift, "A narrative review of the epidemiology, diagnosis, and treatment of latent stress urinary incontinence," Neurourology and Urodynamics, vol. 38, no. 4, pp. S7-S11, 2019.

[4] I. Behravan, S. H. Zahiri, S. M. Razavi, and R. Trasarti, "Finding roles of players in football using automatic particle swarm optimization-clustering algorithm," Big Data, vol. 7, no. 1, pp. 35-56, 2019.

[5] M. M. al-Rifaie, A. Aber, and D. J. Hemanth, "Deploying swarm intelligence in medical imaging identifying metastasis, micro-calcifications and brain image segmentation," IET Systems Biology, vol. 9, no. 6, pp. 234-244, 2015.

[6] X. Wang, X. Xu, J. Luo, Z. Chen, and S. Feng, "Effect of appbased audio guidance pelvic floor muscle training on treatment of stress urinary incontinence in primiparas: a randomized controlled trial," International Journal of Nursing Studies, vol. 104, Article ID 103527, 2021.

[7] O. Celiker Tosun, E. Kaya Mutlu, A. M. Ergenoglu et al., "Does pelvic floor muscle training abolish symptoms of urinary incontinence? A randomized controlled trial," Clinical Rehabilitation, vol. 29, no. 6, pp. 525-537, 2015.

[8] E. T. Reilly, R. M. Freeman, M. R. Waterfield, A. E. Waterfield, P. Steggles, and F. Pedlar, "Prevention of postpartum stress incontinence in primigravidae with increased bladder neck mobility: a randomised controlled trial of antenatal pelvic floor exercises," BJOG, vol. 109, no. 1, pp. 68-76, 2002.

[9] E. Jamard, M. Blouet, T. Thubert, M. Rejano-Campo, R. Fauvet, and A. C. Pizzoferrato, "Utility of 2D-ultrasound in pelvic floor muscle contraction and bladder neck mobility assessment in women with urinary incontinence," J Gynecol Obstet Hum Reprod, vol. 49, no. 1, Article ID 101629, 2020.

[10] L. Chen, D. Luo, X. Yu, M. Jin, and W. Cai, "Predicting stress urinary incontinence during pregnancy: combination of pelvic floor ultrasound parameters and clinical factors," Acta Obstetricia et Gynecologica Scandinavica, vol. 97, no. 8, pp. 966-975, 2018.

[11] M. Fani, R. Salehi, N. Chitsaz, S. Goharpey, and S. Zahednejad, "Transabdominal ultrasound imaging of pelvic floor muscle activity in women with and without stress urinary incontinence: a case-control study," Journal of Obstetrics and Gynaecology Canada, vol. 42, no. 11, pp. 1358-1363, 2020.

[12] M. Hagovská, P. Urdzík, and J. Śvihra, "A randomized interventional parallel study to evaluate the effect of pelvic floor muscle training with stabilization exercises of high and low intensity in women with stress urinary incontinence: the PELSTAB study," Medicine (Baltimore), vol. 99, no. 29, Article ID e21264, 2020.

[13] H. Van Geelen, D. Ostergard, and P. Sand, "A review of the impact of pregnancy and childbirth on pelvic floor function as assessed by objective measurement techniques," International Urogynecol Journal, vol. 29, no. 3, pp. 327-338, 2018.

[14] R. Lu, Y. Zhang, F. R. Dai, and Y. P. Yu, "[Application of transperineal pelvic floor ultrasonography in the diagnosis of female stress urinary incontinence]," Zhonghua Yixue Zazhi, vol. 98, no. 33, pp. 2675-2677, 2018.

[15] K. C. L. Brooks, K. Varette, M. A. Harvey et al., "A model identifying characteristics predictive of successful pelvic floor muscle training outcomes among women with stress urinary incontinence," International Urogynecol Journal, vol. 32, no. 3, pp. 719-728, 2021. 
[16] Y. Yin, Z. Xia, X. Feng, M. Luan, and M. Qin, "Three-dimensional transperineal ultrasonography for diagnosis of female occult stress urinary incontinence," Medical Science Monitor, vol. 25, pp. 8078-8083, 2019.

[17] I. H. Braekken, M. Majida, M. E. Engh, and K. Bø, "Can pelvic floor muscle training reverse pelvic organ prolapse and reduce prolapse symptoms? An assessor-blinded, randomized, controlled trial," American Journal of Obstetrics and Gynecology, vol. 203, no. 2, pp. 170.e1-7, 2010.

[18] M. Ø Nyhus, S. H. Oversand, Ø Salvesen, K. A Salvesen, S. Mathew, and I. Volløyhaug, "Ultrasound assessment of pelvic floor muscle contraction: reliability and development of an ultrasound-based contraction scale," Ultrasound in $\mathrm{Ob}$ stetrics and Gynecology, vol. 55, no. 1, pp. 125-131, 2020.

[19] A. Martan, J. Mašata, and K. Švabík, "Management recidivující stresové inkontinence moči po selhání efektu antiinkontinentních operací [Management of recurrent stress urinary incontinence after anti-incontinence surgery]," Ceská Gynekologie, vol. 82, no. 1, pp. 59-64, 2017. 